\title{
Coronary Computerized Tomography Angiography for Rapid Discharge of Low-Risk Patients with Cocaine-Associated Chest Pain
}

\author{
Kristy M. Walsh, BS ${ }^{a}$, Anna Marie Chang, MDa, Jeanmarie Perrone, MDa, Christine M. McCusker, \\ RN, BSNa, Frances S. Shofer, PhD ${ }^{a}$, Mark J. Collin, BAa, Harold I. Litt, MD, PhD ${ }^{b}$, Judd E. \\ Hollander, $\mathrm{MD}^{a}$
}

aDepartment of Emergency Medicine, Hospital of the University of Pennsylvania, Philadelphia, PA

bDepartment of Radiology, Hospital of the University of Pennsylvania, Philadelphia, PA

\begin{abstract}
Background: Most patients presenting to emergency departments (EDs) with cocaine-associated chest pain are admitted for at least 12 hours and receive a "rule out acute coronary syndrome" protocol, often with noninvasive testing prior to discharge. In patients without cocaine use, coronary computerized tomography angiography (CTA) has been shown to be useful for identifying a group of patients at low risk for cardiac events who can be safely discharged. It is unclear whether a coronary CTA strategy would be efficacious in cocaine-associated chest pain, as coronary vasospasm may account for some of the ischemia. We studied whether a negative coronary CTA in patients with cocaine-associated chest pain could identify a subset safe for discharge.

Methods: We prospectively evaluated the safety of coronary CTA for low-risk patients who presented to the ED with cocaineassociated chest pain (self-reported or positive urine test). Consecutive patients received either immediate coronary CTA in the ED (without serial markers) or underwent coronary CTA after a brief observation period with serial cardiac marker measurements. Patients with negative coronary CTA (maximal stenosis less than 50\%) were discharged. The main outcome was 30-day cardiovascular death or myocardial infarction.

Results: A total of 59 patients with cocaine-associated chest pain were evaluated. Patients had a mean age of $45.6 \pm 6.6$ yrs and were $86 \%$ black, $66 \%$ male. Seventy-nine percent had a normal or nonspecific ECG and $85 \%$ had a TIMI score $<2$. Twenty patients received coronary CTA immediately in the ED, 18 of whom were discharged following CTA (90\%). Thirty-nine received coronary CTA after a brief observation period, with 37 discharged home following CTA (95\%). Six patients had coronary stenosis $\geq 50 \%$. During the 30-day follow-up period, no patients died of a cardiovascular event (0\%; 95\% CI, 0-6.1\%) and no patient sustained a nonfatal myocardial infarction (0\%; 95\% CI, 0-6.1\%).

Conclusions: Although cocaine-associated myocardial ischemia can result from coronary vasoconstriction, patients with cocaineassociated chest pain, a non-ischemic ECG, and a TIMI risk score $<2$ may be safely discharged from the ED after a negative coronary CTA with a low risk of 30-day adverse events.
\end{abstract}

Keywords: cocaine, chest pain, acute coronary syndrome, complications, risk stratification, observation units, computerized tomography

Notes: Dr. Hollander had full access to all the data in the study and takes responsibility for the integrity of the data and the accuracy of the data analysis.

There was no outside funding of any kind used for this study.

Corresponding Author: Judd E. Hollander, MD, Department of Emergency Medicine, Ground Floor, Ravdin Building, Hospital of the University of Pennsylvania, 3400 Spruce Street, Philadelphia, PA 19104-4283. E-mail: hollandj@uphs.upenn.edu 


\section{INTRODUCTION}

Approximately 2.4 million people habitually use cocaine in the United States, making it one of the most widely used illicit drugs [1]. In 2005, cocaine was the most common illicit drug leading to emergency department (ED) visits and accounted for $31 \%$ of all drug-related encounters [2]. Chest pain is a common complication of cocaine use [3], accounting for $16 \%$ of all cocaine-related admissions [4-6]. Six percent of ED patients with cocaine-associated chest pain are diagnosed with acute myocardial infarction (AMI) $[7,8]$. However, identification of the patient with cocaine-associated myocardial infarction is challenging as clinical characteristics may be indistinguishable from those of the cocaine-associated chest pain patient without myocardial infarction [7].

Efforts to risk-stratify patients with cocaine-associated chest pain early in the ED course have been unsuccessful [7,9-11]. Mittleman et al. demonstrated that the greatest risk of AMI is within 60 minutes following cocaine use [12]. Accordingly, clinical studies suggest that most complications occur either before or shortly after ED arrival [5,7]. Based on an analysis of a large retrospective study, it was proposed that patients at risk for complications could be identified within 12 hours of presentation via an observation unit strategy [13]. A 9- to 12-hour observation period has been validated as safe and cost effective if the patient experiences no cardiac complications during that time [10].

Recent data in low-risk patients with chest pain unrelated to cocaine have demonstrated that coronary computerized tomographic angiography (CTA) has high diagnostic accuracy [14-16], is safe [17], and is cost effective [18] for identifying patients for discharge. It is unclear whether these findings can be extended to patients with cocaine-associated chest pain because cocaine can cause AMI in the absence of atherosclerotic coronary disease through coronary vasoconstriction. The purpose of our study was to evaluate the ability of coronary CTA to predict 30-day adverse events in cocaine-associated chest pain and to identify a cohort who can be safely discharged from the ED.

\section{METHODS}

\section{Study Design}

We conducted a prospective cohort study on consecutive patients with cocaine-associated chest pain who received coronary CTA from May 2005 to June 2008 to assess 30-day cardiovascular death and nonfatal myocardial infarction. The study was approved by the University of Pennsylvania Institutional Review Board. This is a substudy of a larger ongoing investigation.

\section{Setting}

The study was conducted in the Hospital of the University of Pennsylvania, which is an urban tertiary referral center with an annual ED census of approximately 55,000 during the enrollment period. The ED has an 8-bed clinical decision/observation unit managed by emergency physicians that can be utilized for evaluation of low- risk chest pain patients. The observation unit chest pain protocol includes serial markers and electrocardiograms (ECG) followed by myocardial perfusion imaging or coronary CTA the next morning. The observation unit is not utilized for admission of chest pain patients on Friday or Saturday, since myocardial perfusion imaging and CTA are not available on Saturday or Sundays.

\section{Selection of Participants}

Patients who presented to the ED with a chief complaint of chest discomfort subsequent to cocaine use (self-reported or positive urine test) that prompted an ECG for evaluation of a potential acute coronary syndrome were eligible for inclusion if the ECG did not show acute ischemia, the emergency physician was concerned enough to plan to admit the patient to rule out an acute coronary syndrome, and the patient received a coronary CTA. We did not require urine testing when patients self-reported cocaine use, since it is our experience that when patients actually report cocaine use, they are seldom incorrect.

Patients were excluded if they had chest pain explained by local trauma, radiographic abnormalities, or they were at such low risk that they would otherwise be discharged home without admission or a provocative test to exclude an acute coronary syndrome. High-risk patients were defined as those with an initial ECG diagnostic of ischemia or acute myocardial infarction, transient ST-segment elevation or depression $\geq 1 \mathrm{~mm}$ that persisted for at least 1 minute, elevated cardiac markers, recurrent ischemic chest pain, or hemodynamic instability. These patients were admitted to the hospital, did not receive a coronary CTA, and were excluded from analysis. Patients were also excluded if they had a contraindication to coronary CTA (such as iodinated contrast allergy) or if coronary CTA was not available. Coronary CTA was available during weekday daytime hours and approximately every third weekend when a certified radiologist was present. Therefore, patients who presented outside of these hours could only be enrolled after an overnight stay in the observation unit. All patients with a history of cocaine use who received a coronary CTA were included in the analysis regardless of subsequent clinical course.

\section{DATA COLLECTION AND PROCESSING}

\section{Initial Evaluation}

Structured data collection was performed prospectively by the treating physician using a closed question data instrument that was in accordance with the Standardized Reporting Guidelines [19] and Key Definitions [20] and included self-reported demographic characteristics, cardiac risk factors, chest pain characteristics, associated symptoms, medications, and initial vital signs, ECG, treatment, and disposition.

\section{Coronary CTA}

The protocol for coronary CTA included patients with a TIMI risk score of 0-2 (approximately $50-70 \%$ of patients being evaluated 
for ACS) [21-22]. All patients provided verbal consent to receive coronary CTA according to standard radiology department procedure. Patients with tachycardia were treated with benzodiazepines or diltiazem at the discretion of the emergency physician caring for the patient. Beta-blockers, which are usually used for heart rate control in coronary CTA, were not used because of the risk of unopposed alpha-agonist action in patients using cocaine. Coronary CTA was performed using predominantly 64-slice or dual-source scanners (Siemens Medical Solutions, Malvern, PA). The study began with a low-dose, noncontrast, ECG-triggered acquisition through the entire chest for the purpose of calcium scoring and evaluation of lung abnormalities. This was followed by a weight-based intravenous injection of $80-120 \mathrm{~mL}$ of nonionic iodinated contrast (Omnipaque 350, GE Amersham, Milwaukee, WI) with bolus tracking in the descending aorta. After the appropriate scan delay, an ECG-gated acquisition from the pulmonary artery bifurcation through the inferior heart border was performed. Studies were reviewed on dedicated 3D workstations (Leonardo and MMWP, Siemens Medical Solutions, and AdvantageWindows 4.2, GE) using axial, multiplanar reformatted, and thin-slab, maximum-intensity projection images in an interactive display. Image data were reconstructed at multiple phases of the cardiac cycle, and postprocessed and analyzed on independent workstations. The degree of any observed stenosis was measured with an electronic caliper by comparing the lumen diameter with the diameter of a proximal reference segment [23]. Studies were read by attending radiologists with subspecialty cardiovascular imaging training; all met ACC/AHA level 3 training guidelines for cardiac CT. Interpretations were provided for all studies. Study quality and technical limitations were reported in the reading, as well as any regions of coronary arteries that could not be interpreted. Management of these limitations was left to the treating physician. For clinical purposes, during the study period, the coronary CTA was considered positive if the patient had $\mathrm{a} \geq 70 \%$ stenosis of the right coronary, left main, left anterior descending, or circumflex arteries or their first-order branches, and admission was recommended. The coronary CTA was considered negative if the patient had a $<50 \%$ stenosis, and no further evaluation was recommended for potential acute coronary syndrome. In patients with intermediate grades of stenosis (50-69\%) or a calcium score greater than 100, further evaluation was performed at physician discretion.

\section{Hospital Course}

Patients with a negative coronary CTA were discharged from the ED without further cardiac markers or observation, unless other medical problems warranted admission. Patients with a positive coronary CTA were admitted to the hospital and followed daily for cardiovascular complications including death, myocardial infarction [24], heart failure, and dysrhythmias. For all patients, cardiac markers were obtained at time of entrance into the ED; for admitted patients, serial cardiac markers were obtained at least 6-12 hours after presentation. During the study period, we used an enzyme-linked immunoassay for cardiac troponin I and CK-MB
(Abbott AxSYM automated analyzer; Abbott Laboratories, Mountain View, CA). Levels were considered elevated if any measurement exceeded the institutional threshold for normal (CK-MB $>5$ $\mathrm{mg} / \mathrm{dL}$ and cardiac troponin $\mathrm{I}>0.4 \mathrm{mg} / \mathrm{dL}$ ).

Patients with abnormal coronary CTA without evidence of myocardial necrosis or rest ischemia underwent graded exercise testing using the modified Bruce Protocol or pharmacological stress imaging, if ordered by the treating physician. A positive stress test was defined as ST-segment elevation or depression of $>1 \mathrm{~mm}$ or reversible ischemia with or without chest discomfort during the test.

\section{Follow-up}

At the time of presentation, all patients were asked to provide the phone numbers of their homes, businesses, and a relative. Alternative contact information such as pager numbers and e-mail were also obtained, when applicable. Patients were contacted at least 30 days after discharge and specifically questioned about recurrent chest pain, nonfatal myocardial infarction, and repeat hospitalization. A 30-day timeframe was chosen as the primary outcome based upon consensus recommendation of major cardiology and emergency medicine societies [19]. If the patients or secondary contacts were unavailable, medical records were reviewed for repeat visits. For patients in whom these methods failed to provide survival information, we searched the Social Security Death Index (http://ssdi.rootsweb.ancestry.com/) on August 13, 2008. All reported adverse events (myocardial infarction, death) were confirmed by records review.

\section{Data Analysis}

Our main outcome was 30-day death or nonfatal myocardial infarction. Data are presented descriptively as the percent frequency occurrence with 95\% confidence intervals. Using a binomial distribution, Clopper-Pearson confidence intervals were calculated with StatXact 6.1 (Cytel Software Corporation, Cambridge, MA).

\section{RESULTS}

\section{Characteristics of Study Subjects}

There were 59 patients with cocaine-associated chest pain identified who received coronary CTA. Patients had a mean age of $46 \pm 6.6$ years and were predominantly black (86\%), male $(66 \%)$, and tobacco smokers (69\%). When evaluated for traditional risk factors of coronary artery disease, 19 (32\%) had a history of hypertension, and 12 (20\%) had diabetes. Other risk factors were less common (Table 1). The most common chest pain description was pressure/tightness (51\%), and 33 (56\%) experienced shortness of breath. Seventy-nine percent of patients had a normal or nondiagnostic ECG, and most patients had a TIMI risk score of 0 or 1 (85\%). Demographic, historical, and presenting characteristics of the study population are presented in Table 1. 
Table 1: Demographic, Historical, and Presenting Characteristics of Study Patients

\begin{tabular}{lc}
\hline Characteristic & $\begin{array}{c}\text { Number (\%) } \\
\mathbf{n = 5 9}\end{array}$ \\
\hline Age (mean \pm SD) & $45.6 \pm 6.6$ \\
\hline Male (\%) & $39(66 \%)$ \\
\hline Race & $49(86 \%)$ \\
\hline Black & $8(14 \%)$ \\
\hline Caucasian & $2(3 \%)$ \\
\hline Other/unknown & $19(32 \%)$ \\
\hline Cardiac risk ractors & $4(7 \%)$ \\
\hline Hypertension & $10(18 \%)$ \\
\hline Hypercholesterolemia & $12(20 \%)$ \\
\hline Family history of CAD & $41(69 \%)$ \\
\hline Diabetes mellitus & $51(86 \%)$ \\
\hline Current tobacco use &
\end{tabular}

Number of cardiac risk factors

\begin{tabular}{lc}
0 & $9(16 \%)$ \\
1 & $23(40 \%)$ \\
2 & $17(30 \%)$ \\
3 & $5(5 \%)$ \\
\hline 4 & $3(5 \%)$
\end{tabular}

Past medical problems

\begin{tabular}{lc} 
Undiagnosed chest pain & $11(19 \%)$ \\
Myocardial infarction & $5(8 \%)$ \\
\hline Congestive failure & $0(0 \%)$ \\
\hline Arrhythmias & $1(2 \%)$
\end{tabular}

\section{Chest pain characteristics}

Location

\begin{tabular}{lc}
\hline Substernal & $29(49 \%)$ \\
\hline Left chest or arm & $20(34 \%)$ \\
\hline Right sided & $6(10 \%)$ \\
\hline Epigastrum & $0(0 \%)$ \\
\hline Other/unknown & $4(7 \%)$ \\
\hline Quality & $30(51 \%)$ \\
\hline Crushing/pressure/tightness & $13(22 \%)$ \\
\hline Sharp/stabbing
\end{tabular}

\section{Clinical Course}

Of the 59 patients who received coronary CTA, 20 received it in the ED prior to serial cardiac markers. These patients had a median length of stay from triage until ED disposition of 7.7 hours (interquartile range, 5.9-11.7 hours). Of the 20 patients who re-

\begin{tabular}{lc} 
Aching/dull & $5(8 \%)$ \\
\hline Burning/indigestion & $3(5 \%)$ \\
\hline Other/unknown & $8(14 \%)$ \\
\hline Associated symptoms & \\
\hline Shortness of breath & $33(56 \%)$ \\
\hline Diaphoresis & $15(25 \%)$ \\
\hline Palpitations & $7(12 \%)$ \\
\hline Nausea & $11(19 \%)$ \\
\hline Vomiting & $3(5 \%)$ \\
\hline Syncope & $2(3 \%)$
\end{tabular}

\section{Vital Signs at Presentation}

Systolic blood pressure

$\begin{array}{lc}>139 \mathrm{~mm} \mathrm{Hg} & 21(36 \%) \\ >91-139 \mathrm{~mm} \mathrm{Hg} & 38(64 \%) \\ <90 \mathrm{~mm} \mathrm{Hg} & 0(0 \%)\end{array}$

Diastolic blood pressure

$>90 \mathrm{~mm} \mathrm{Hg} 10(17 \%)$

Pulse

$\begin{array}{lc}>100 \text { beats/minute } & 10(17 \%) \\ 60-100 \text { beats/minute } & 48(81 \%) \\ <60 \text { beats/minute } & 1(2 \%)\end{array}$

Respiratory rate

$>25$ per minute 2 (3\%)

10-25 per minute $\quad 57(97 \%)$

Temperature: $>38 \mathrm{C} \quad 0(0 \%)$

Presenting electrocardiogram

\begin{tabular}{lc}
\hline Normal & $31(53 \%)$ \\
\hline Nonspecific & $15(26 \%)$ \\
\hline Non-diagnostic & $7(12 \%)$ \\
\hline Early repolarization & $3(5 \%)$ \\
\hline Ischemia known to be old & $2(3 \%)$ \\
\hline Ischemia not known to be old & $0(0 \%)$ \\
\hline Diagnostic of acute myocardial infarction & $0(0 \%)$ \\
\hline TIMI Risk Score & $30(51 \%)$ \\
\hline 0 & $20(34 \%)$ \\
\hline 1 & $9(15 \%)$ \\
\hline 2 & $0(0 \%)$ \\
\hline 3 or 4 & \\
\hline
\end{tabular}

ceived coronary CTA in the ED, 18 (90\%) were discharged home after coronary CTA, avoiding admission to the observation unit or the hospital. Two patients with coronary CTAs done in the ED were admitted for further testing (Table 2). 
Table 2: Clinical Course of Patients with Any Coronary Disease Noted on CTA

\begin{tabular}{|c|c|c|c|c|c|c|c|c|c|c|c|}
\hline \multirow[b]{2}{*}{ Characteristics at Presentation } & \multicolumn{4}{|c|}{ ED course } & \multicolumn{7}{|c|}{ CTA Results } \\
\hline & EKG & TIMI & Treatment & Disposition & $\begin{array}{l}\text { Calcium } \\
\text { score }\end{array}$ & $\begin{array}{l}\text { L. } \\
\text { Main }\end{array}$ & LAD & $\begin{array}{l}\text { L. } \\
\text { Circ }\end{array}$ & $\begin{array}{l}\text { RCA/ } \\
\text { PDA }\end{array}$ & $\begin{array}{c}\text { Hospital } \\
\text { Course }\end{array}$ & $\begin{array}{c}\text { 30-day } \\
\text { F/U }\end{array}$ \\
\hline $\begin{array}{l}\text { 54-year-old B M tobacco smoker } \\
\text { with HTN, high cholesterol, FH of } \\
\text { heart disease, and a prior negative } \\
\text { stress test; substernal CP at rest }\end{array}$ & $\begin{array}{l}\text { Nonspecific } \\
\text { (T wave } \\
\text { flattening } \\
\text { in inferior } \\
\text { leads) }\end{array}$ & 2 & ASA & $\begin{array}{l}\text { Presented } \\
\text { late night; } \\
\text { kept in ED } \\
\text { for morning } \\
\text { CTA }\end{array}$ & 302.6 & 0 & 50 & 60 & 50 & $\begin{array}{l}\text { Uneventful } \\
\text { hospital } \\
\text { course w/o } \\
\text { further } \\
\text { testing }\end{array}$ & $\begin{array}{c}\text { No } \\
\text { events }\end{array}$ \\
\hline $\begin{array}{l}\text { 44-year-old B F tobacco smoker } \\
\text { with } \mathrm{HTN}, \mathrm{DM} \text {, and a FH of } \\
\text { heart disease and no prior tests; } \\
\text { crushing right-sided radiating } \\
\mathrm{CP} \text { at rest }\end{array}$ & Normal & 2 & ASA & $\begin{array}{l}\text { CDU with } \\
\text { CTA in CDU } \\
\text { after serial } \\
\text { markers }\end{array}$ & 0.7 & 0 & 50 & 0 & 0 & $\begin{array}{l}\mathrm{D} / \mathrm{C} \text { home } \\
\text { from CDU }\end{array}$ & $\begin{array}{c}\text { No } \\
\text { events }\end{array}$ \\
\hline $\begin{array}{l}\text { 47-year-old B M tobacco smoker } \\
\text { with high cholesterol and no } \\
\text { prior tests; pleuritic left sided } \\
\text { radiating CP at rest }\end{array}$ & Normal & 2 & $\begin{array}{c}\text { ASA, } \\
\text { nitrates }\end{array}$ & $\begin{array}{l}\text { CDU with } \\
\text { CTA in CDU } \\
\text { after serial } \\
\text { markers }\end{array}$ & 0.7 & 0 & 60 & 30 & 0 & $\begin{array}{l}\text { Had exercise } \\
\text { sestamibi } \\
\text { that was } \\
\text { negative. } \\
\text { D/C home } \\
\text { from CDU. }\end{array}$ & $\begin{array}{l}\text { Unable } \\
\text { to obtain } \\
\text { 30-day } \\
\text { F/U }\end{array}$ \\
\hline $\begin{array}{l}\text { 44-year-old B M tobacco smoker } \\
\text { with HTN, and no prior tests; } \\
\text { nonpleuritic burning left-sided } \\
\text { radiating CP not at rest }\end{array}$ & $\begin{array}{l}\text { Nonspecific } \\
\text { (isolated T wave } \\
\text { flattening in III) }\end{array}$ & 0 & $\begin{array}{c}\text { ASA, } \\
\text { nitrates }\end{array}$ & $\begin{array}{l}\text { Telemetry } \\
\text { after } \\
\text { immediate } \\
\text { CTA }\end{array}$ & 0 & 0 & 40 & 40 & 60 & $\begin{array}{l}\text { Uneventful } \\
\text { hospital } \\
\text { course w/o } \\
\text { further } \\
\text { objective } \\
\text { testing }\end{array}$ & $\begin{array}{c}\text { No } \\
\text { events }\end{array}$ \\
\hline $\begin{array}{l}45 \text {-year-old B F tobacco smoker } \\
\text { with no prior tests; exertional } \\
\text { aching substernal CP }\end{array}$ & Normal & 0 & ASA & $\begin{array}{l}\text { CDU with } \\
\text { CTA in CDU } \\
\text { after serial } \\
\text { markets }\end{array}$ & 0 & 0 & 50 & 0 & 0 & $\begin{array}{l}\mathrm{D} / \mathrm{C} \text { home } \\
\text { from CDU }\end{array}$ & $\begin{array}{c}\text { No } \\
\text { events }\end{array}$ \\
\hline $\begin{array}{l}\text { 48-year-old B F tobacco smoker } \\
\text { with HTN, FH of heart disease, } \\
\text { prior stress test and cardiac cath } \\
\text { with unknown results; crushing }\end{array}$ & $\begin{array}{l}\text { Left ventricular } \\
\text { hypertrophy } \\
\text { without change } \\
\text { from old }\end{array}$ & 2 & $\begin{array}{c}\text { ASA, } \\
\text { Nitrates }\end{array}$ & $\begin{array}{l}\text { CDU with } \\
\text { CTA in CDU } \\
\text { after serial } \\
\text { markers }\end{array}$ & 147 & 0 & 60 & 0 & 0 & $\begin{array}{l}\text { Adm to TELE } \\
\text { for cardiac } \\
\text { cath (showed } \\
70 \% \text { LAD); } \\
\text { managed } \\
\text { medically, } \\
\text { D/C day } 3\end{array}$ & $\begin{array}{c}\text { No } \\
\text { events }\end{array}$ \\
\hline
\end{tabular}

$A C S$, acute coronary syndrome; $A d m$, admit; $B$, Black; $C A D$, coronary artery disease; $C D U$, clinical decision unit; $C P$, chest pain; $D / C$, discharged; $D M$, diabetes mellitus; F, female; FH, family history; F/U, follow-up; HTN, hypertension; M, male; TELE, telemetry

The remaining 39 patients received the coronary CTA while in the observation unit, in conjunction with serial markers. These patients had a median length of stay from triage until transfer to observation unit of 5.6 hours (interquartile range, 4.1-7.7 hours). Following coronary CTA, 37 of the 39 were discharged home (95\%). One patient with a positive coronary CTA was admitted to the hospital, where cardiac catheterization found similar results. The patient was managed medically (Table 2).

Overall, 6 patients were identified who had underlying coronary artery disease. The clinical course and outcome of these 6 patients is summarized in Table 2. The only one of these patients lost to follow-up had a stress test that was negative for reversible ischemia prior to discharge from the hospital.

\section{Main Clinical Outcomes}

Thirty-day follow-up by direct contact or record review was obtained for 49 of the 59 enrolled (83\%); the main outcome was death or nonfatal AMI. No patient described as low risk (normal or nonspecific ECG, negative cardiac markers, TIMI risk score $\leq 2$ ) and discharged after a negative coronary CTA experienced an adverse cardiac event over the 30-day follow-up period (95\% confidence interval, 0-6.1\%). The remaining 10 patients did not appear in the Social Security Death Index and no 
deaths or cardiovascular complications were detected through record review.

\section{Coronary CTA Results}

Forty-seven patients had a calcium score of 0,10 had a score of $1-100$, and 2 had a score $>100$. Coronary CTA found that 53 patients $(90 \%)$ had a maximal stenosis $<50 \%$, with 45 having no coronary disease. There were 6 patients with a stenosis of $50 \%$ or greater. Details of the clinical course, further evaluation, and 30day outcomes of patients with any coronary disease detected on coronary CTA are shown in Table 2.

\section{DISCUSSION}

Recent literature suggests coronary CTA as a useful approach to evaluate low-risk patients with potential ACS unrelated to cocaine. In a study of 568 low-risk ED patients with chest pain, those with negative coronary CTA did not have any adverse outcomes (nonfatal AMI or death) within 30 days of the index ED visit [25]. Prior studies have shown that coronary CTA has high diagnostic accuracy (82-100\% sensitivity, 82-98\% specificity, negative predictive value of 95-100\%) in low-risk patients for detection of coronary artery disease [26-34]. Coronary CTA has been shown to be as safe [17] and more cost effective [18] than other methods of evaluating these patients. In a cost analysis of four strategies for the evaluation of chest pain, Chang et al. verified that coronary CTA was the least expensive strategy and resulted in the shortest length of stay with both a decreased rate of admission and decreased rate of return visits in patients with possible acute coronary syndrome [18].

This approach, however, has not been evaluated in patients with a history of cocaine use. In this study of ED patients with cocaine-associated chest pain, we found that low-risk patients identified by a non-ischemic ECG and a TIMI risk score $\leq 2$ may be safely discharged after a negative CTA without consequence of a nonfatal MI or death at 30 days.

Cocaine-associated myocardial infarction occurs due to several well-studied pathophysiologic effects of cocaine. Many patients (67\%) with cocaine-associated MI have underlying atherosclerotic coronary artery disease [35]. Autopsy reports in patients with cocaine-associated fatal myocardial infarction found atherosclerosis in 50-63\% of young patients [36-38]. Evidence of coronary artery disease in young patients may imply a relationship between cocaine use and premature atherosclerosis; in one study, the average age of cocaine-induced AMI patients was 38 years old [35]. The finding of accelerated atherosclerosis secondary to cocaine has also been demonstrated in animal models [39]. This is particularly important because, while cocaine-induced vasoconstriction can occur in atherosclerotic affected or unaffected coronary artery segments, the effects of cocaine use are magnified in diseasesd segments [40]. In this study, coronary CTA identified 6 patients with underlying coronary artery disease despite the low-risk nature of the patient population. Significant coronary artery disease is associated with adverse long-term prognosis. Cocaine is also well known to cause myocardial ischemia [41] even in patients with patent coronary arteries. Cocaine can increase oxygen demand while decreasing blood flow through coronary vasoconstriction $[13,42-43]$, increase platelet aggregation [36,44-45], and thrombosis $[37,46-48]$. Although the greatest risk for cocaine-induced AMI is within the first hour following use $[7,12]$, delayed or recurrent coronary vasoconstriction can occur concomitantly with the rise in concentrations of cocaine metabolites (benzoylecgonine and ecgonine methyl ester) more than 1 hour after cocaine use [49]. In our study, most CTAs were performed after serial cardiac markers and a period of observation; however, 18 patients were discharged after an "immediate" CTA and we did not observe any adverse effects. However, this is a small subset of patients, and it is perhaps still advisable to use serial markers in those patients who present with cocaine-associated chest pain.

The current American College of Cardiology/American Heart Association (ACC/AHA) recommendations include a 9- to 12-hour observation period followed by stress testing for cocaine-induced chest pain [50]. Stress tests can be used to assess low- and moderate-risk patients with cocaine-associated chest pain. Kontos et al. found that of 211 patients with a negative test, no patient suffered an AMI [51]. Their study implies that along with low clinical suspicion and a non-ischemic ECG, a negative perfusion imaging test can identify patients who may be safely discharged without occurrence of cardiac events at 30 days. A case series by Littmann et al. found that early stress testing (1-2 weeks after cocaine use) can lead to false-positive results with further unnecessary and costly testing [52]. They suggested that clinically-stable patients with cocaineassociated chest pain wait 2-3 weeks after cocaine use to be evaluated [52]. Weber et al. found that there was no difference in 30-day outcomes in patients who received inpatient or outpatient stress tests following cocaine use [53]. However, Manini et al. reported that inpatient testing is important, as $37 \%$ of patients who received a referral for outpatient cardiac testing were noncompliant [54], and this rate may even be lower in patients with substance abuse. Exercise stress testing, commonly used in patients with symptoms unrelated to cocaine [55], appears to perform similarly in patients with cocaine-associated chest pain, but nonetheless lacks high levels of diagnostic accuracy in both patient populations.

Accordingly, our results imply a role for CTA in screening lowrisk, cocaine-associated chest pain patients to exclude AMI and identify significant coronary stenosis. Furthermore, it may be more advantageous than other imaging modalities because it yields more diagnostic information. Although AMI is the chief concern in evaluating patients with cocaine-associated chest pain, cocaine can also cause pneumothorax, aortic dissection, pneumonia, "crack lung," and pulmonary embolism, all of which may be detected by CTA. Other cardiac imaging modalities such as exercise stress testing will not identify these findings, thus cannot screen for these other potential diagnoses.

It is possible that early evaluation of patients with cocaineassociated chest pain may be a tool to identify cocaine-mediated coronary vasoconstriction while cocaine metabolites are still present, even in patients with patent coronary arteries, which 
may be more analogous to the clinical setting of cocaine-mediated vasoconstriction. This concept needs more exploration as the image speed and resolution of CTA improves.

Coronary CTA is advantageous in the ED setting because it can be completed quickly, with final imaging results available shortly thereafter [56]. With implementation of dose-reduction protocols [57] that selectively utilize maximal x-ray exposure only during important phases of the cardiac cycle, radiation exposure becomes similar to nuclear cardiology examination [58]. Lower contrast volumes used in CTA reduce the likelihood of nephrotoxicity, which is especially important if findings warrant further evaluation by coronary angiography [56].

\section{LIMITATIONS}

There are several potential limitations to our study design that merit discussion. Due to the sample size, the upper limit of the confidence interval for 30-day adverse events is still approximately $6 \%$. Although our data suggest that coronary CTA holds promise, additional study is warranted before its safety can be confirmed. All patients were evaluated at a single urban university hospital ED, which raises concerns regarding the ability to generalize to other regions and patient populations.

Our study may have been subject to some selection bias. We relied on patient self-reported cocaine use or a positive urine screen to identify cocaine-using patients; we cannot be certain we included all patients with cocaine-associated chest pain. Furthermore, those screening positive may not have received a CTA if they were evaluated at times other than the hours when CTA technology was available. No attempt was made to ascertain the chronicity or route of cocaine use, thus we cannot determine any association between presence of coronary artery disease and duration of cocaine use. In addition, screening for other drugs was not performed, thus the concomitant effects of polysubstance use could not be measured.

It is also important to note that we did not perform a direct comparison of coronary CTA to other methods of evaluating patients with cocaine-associated chest pain, such as stress test or myocardial perfusion imaging. As such, we cannot directly state which strategy might be preferable.

There are theoretical reasons why coronary CTA may not perform as well in the setting of cocaine use. Cocaine causes tachycardia, and CTA images are dependent on heart rate. However, most patients in this study and prior studies of patients with cocaine-associated chest pain are not tachycardic $[7,8,10,59]$. There are some patients with tachycardia after cocaine use who may not be candidates for coronary CTA because most protocols for noncocaine-using patients require administration of betaadrenergic blockers for heart rate control, and these agents should be avoided in the setting of cocaine $[41,50]$. Use of CT scanners with higher temporal resolution, such as the dual source machine used later in our study, may relax the requirement for strict heart rate control; however, our sample was too small to evaluate any effects of this technology.

\section{CONCLUSIONS}

Our initial experience with coronary CTA in low-risk patients with cocaine-associated chest pain suggests that it may have clinical utility in identifying patients eligible for safe and rapid discharge. In this initial evaluation, we found that low-risk patients as defined by non-ischemic EKG, a TIMI risk score $<2$, and a negative CTA can be safely discharged without risk of nonfatal MI or death at 30 days. It should be noted, however, that the upper limit of our confidence interval is about $6 \%$. Therefore, further studies should be conducted before implementation of this approach is widely accepted.

The authors have no potential financial conflicts of interest to report.

\section{REFERENCES}

1. Substance Abuse and Mental Health Services Administration, Office of Applied Studies. Results from the 2006 National Survey on Drug Use and Health: National Findings. NSDUH Series H-32, DHHS Publication No. SMA 07-4293. Rockville, MD, 2007.

2. Substance Abuse and Mental Health Services Administration, Office of Applied Studies. Drug Abuse Warning Network, 2005: National Estimates of Drug-Related Emergency Department Visits. DAWN Series D-29, DHHS Publication No. SMA 07-4256, Rockville, MD, 2007.

3. Minor RL, Scott BD, Brown DD, Winniford MD. Cocaineinduced myocardial infarction in patient with normal coronary arteries. Ann Intern Med 1999;115:797-806.

4. Albertson TE, Marelich GP, Tharratt RS. Cocaine. In: Haddad LM, Shannon MW, Winchester JF, eds. Clinical management of poisoning and drug overdose, 3rd ed. Philadelphia: WB Saunders Company, 1998: 542-551.

5. Brody SL, Slovis CM, Wrenn KD. Cocaine-related medical problems: consecutive series of 233 patients. Am J Med 1990;88:325-331.

6. Derlet RW, Albertson TE: Emergency department presentation of cocaine intoxication. Ann Emerg Med. 1989;18: 182-186.

7. Hollander JE, Hoffman RS, Gennis P, et al. Prospective multicenter evaluation of cocaine-associated chest pain. Acad Emerg Med 1994;1:330-339.

8. Weber JE, Chundnofsky CR, Boczar M, et al. Cocaine associated chest pain: how common is myocardial infarction? Acad Emerg Med 2000;7:873-877.

9. Hollander JE, Levitt A, Young GP, et al. Effect of recent cocaine use on the specificity of cardiac markers for diagnosis of acute myocardial infarction. Am Heart J 1998;135: 245-252.

10. Weber JE, Shofer FS, Larkin GL, et al. Validation of a brief observation period for patients with cocaine-associated chest pain. N Engl J Med 2003;348:510-517. 
11. Chase M, Brown AM, Robey JL, et al. Application of the TIMI risk score in ED patients with cocaine-associated chest pain. Am J Med 2007;25:1015-1018.

12. Mittleman MA, Mintzer D, Maclure M, et al. Triggering of myocardial infarction by cocaine. Circulation

1999;99:2737-2741.

13. Hollander JE, Hoffman RS, Burstein JL, et al. Cocaine Associated Myocardial Infarction Study Group. Cocaine associated myocardial infarction: mortality and complications. Arch Intern Med 1995;155:1081-1086.

14. Budoff MJ, Achenbach S, Duerinckx A. Clinical utility of computed tomography and magnetic resonance techniques for noninvasive coronary angiography. J Am Coll Cardiol 2003;42:1867-1878.

15. Ropers D, Baum U, Pohle K, et al. Detection of coronary artery stenoses with thin-slice multi-detector row spiral computed tomography and multiplanar reconstruction. Circulation 2003;107:664-666.

16. Nieman K, Cademartiri F, Lemos PA, et al. Reliable noninvasive coronary angiography with fast submillimeter multislice spiral computed tomography. Circulation 2002;106:2051-2054.

17. Hoffman U, Nagurney JT, Moselewski F, et al. Coronary multidetector computed tomography in the assessment of patients with acute chest pain. Circulation 2006;114:2251-2260.

18. Chang AM, Shofer FS, Weiner MG, et al. Actual financial comparison of four strategies to evaluate patient with potential acute coronary syndromes. Acad Emerg Med 2008;15:649-655.

19. Hollander JE, Blomkalns AL, Brogan GX, et al. Standardized reporting guidelines for studies evaluating risk stratification of emergency department patients with potential acute coronary syndromes. Ann Emerg Med 2004;44:589-598.

20. Cannon CP, Battler A, Brindis RG, et al. American College of Cardiology key data elements and definitions for measuring the clinical management and outcomes of patients with acute coronary syndromes. A report of the American College of Cardiology Task Force on Clinical Data Standards (Acute Coronary Syndromes Writing Committee). J Am Coll Cardiol 2001;38: 2114-2130.

21. Pollack CV Jr, Sites FD, Shofer FS, et al. Application of the TIMI risk score for unstable angina and non-ST elevation acute coronary syndrome to an unselected emergency department chest pain population. Acad Emerg Med 2006;13:13-18.

22. Chase M, Robey JL, Zogby KE, et al. Prospective validation of the TIMI risk score in the emergency department chest pain patient population. Ann Emerg Med 2006;48:252-259.

23. Hoffmann MH, Shi H, Schmitz BL, et al. Noninvasive coronary angiography with multislice computed tomography. JAMA 2005;293(20):2471-2478.

24. Alpert JS, Thygesen K, Antman E, et al. Myocardial infarction redefined-a consensus document of The Joint European Society of Cardiology/American College of Cardiology Committee for the redefinition of myocardial infarction. J Am Coll Cardiol 2000;36:959-969.
25. Hollander J, Chang AM, McCusker C, et al. Coronary computerized tomography for rapid discharge of low risk patients with potential acute coronary syndromes. Acad Emerg Med 2008;15 (5 suppl):S10-11.

26. Gallagher MJ, Ross MA, Raff GL, et al. The diagnostic accuracy of 64-slice CT coronary angiography compared with stress nuclear imaging in emergency department low risk chest pain patients. Ann Emerg Med 2007;2:125-36.

27. McLaughlin VV, Balogh T, Rich S. Utility of electron beam computed tomography to stratify patients presenting to the emergency room with chest pain. Am J Cardiol 1999;84:327-328.

28. Laudon DA, Vukov LF, Breen JF, et al. Use of electron beam computed tomography in the evaluation of chest pain patients in the emergency department. Ann Emerg Med 1998;33:15-21.

29. Georgiou D, Budoff MJ, Kaufer E, et al. Screening patients with chest pain in the emergency department using electron beam tomography: a follow-up study. J Am Coll Cardiol 2001;38:105-10.

30. Goldstein JA, Gallagher MJ, O’Neill WW, et al. A randomized controlled trial of multi-slice coronary computed tomography for evaluation of acute chest pain. J Am Coll Cardiol 2007;49:863-871.

31. Achenbach S. Computed tomography coronary angiography. J Am Coll Cardiol 2006;38:1919-1928.

32. Rubinshtein R, Halon DA, Gaspar T, et al. Usefulness of 64-slice cardiac computed tomographic angiography for diagnosing acute coronary syndromes and predicting clinical outcome in emergency department patients with chest pain of uncertain origin. Circulation. 2007;115:1762-1768.

33. Limkakeng AT, Halpern E, Takakuwa KM. Sixty four-slice multidetector computed tomography: the future of ED cardiac care. Am J Emerg Med 2007;25:450-458.

34. Schussler JM, Smith ER. Sixty-four-slice computed tomographic coronary angiography: will the "triple rule out" change chest pain evaluation in the ED? Am J Emerg Med 2007; 25:367-375.

35. Hollander JE, Shih RD, Hoffman RS, et al. Predictors of coronary artery disease in patients with cocaine associated myocardial infarction. Cocaine-Associated Myocardial Infarction (CAMI) Study Group. Am J Med 1997;102:158-163.

36. Mittleman RE, Wetli CV. Cocaine and sudden "natural" death. J Forensic Sci 1987;32:11-19.

37. Dressler FA, Malekzadeh S, Roberts WC. Quantitative analysis of amounts of coronary arterial narrowing in cocaine addicts. Am J Cardiol 1990;65:303-308.

38. Tardiff K, Gross E, Wu J, et al. Analysis of cocaine-positive fatalities. J Forensic Sci 1989;34:53-63.

39. Arnett EN, Isner JM, Redwood DR, et al. Coronary artery narrowing in coronary heart disease: comparison of cineangiographic and necropsy findings. Ann Intern Med 1979;91:350-645.

40. Flores ED, Lange RA, Cigarroa RG, et al. Effect of cocaine on coronary artery dimensions in atherosclerotic coronary 
artery disease: enhanced vasoconstriction at sites of significant stenoses. J Am Coll Cardiol 1990;16:74-79.

41. Hollander JE, Hoffman RS. Cocaine-induced myocardial infarction: an analysis and review of the literature. J Emerg Med 1992;10:169-177.

42. Kumar K, Holden WE. Drug-induced pulmonary vascular disease: mechanisms and clinical patterns. West J Med 1986;145: 343-349.

43. Lange RA, Cigarroa RG, Yancy CW, et al. Cocaine-induced coronary artery vasoconstriction. N Eng J Med 1989;321: 1557-1562.

44. Togna G, Tempesta E, Togna AR, et al. Platelet responsiveness and biosynthesis of thromboxane and prostacyclin in response to in vitro cocaine treatment. Haemostasis 1985;15: 100-107.

45. Isner JM, Estes M, Thompson PD, et al. Acute cardiac events temporally related to cocaine abuse. N Eng J Med 1985;315: 1438-1443.

46. Stenberg RG, Winniford MD, Hillis LD, et al. Simultaneous acute thrombosis of two major coronary arteries following intravenous cocaine use. Arch Pathol Lab Med 1989;113:521-524.

47. Moliterno DJ, Lange RA, Gerard RD, et al. Influence of intranasal cocaine on plasma constituents associated with endogenous thrombosis and thrombolysis. Am J Med 1994;96:492-496.

48. Weber JE, Hollander JE, Murphy SA, et al. Quantitative comparison of coronary artery flow and myocardial perfusion in patients with acute myocardial infarction in the presence and absence of recent cocaine use. J Thromb Thrombolysis 2002:14(3):239-245.

49. Brogan WC III, Lange RA, Glamann B, et al. Recurrent coronary vasoconstriction caused by intranasal cocaine: possible role for metabolites. Ann Intern Med 1992;116:556-561.

50. McCord J, Hani J, Hollander JE, et al. Management of cocaine-associated chest pain and myocardial infarction: a scientific statement from the American Heart Association Acute
Cardiac Care Committee of the Council on Clinical Cardiology. Circulation 2008;117:1897-1907.

51. Kontos MC, Schmidt KL, Nicholson CS, et al. Myocardial perfusion imaging with technetium-99m sestamibi in patients with cocaine-associated chest pain. Ann Emerg Med 1999;33(6): 639-645.

52. Littman L, Miller RF, Monroe MH. Stress testing in patients with cocaine-associated chest pain. J Emerg Med 2004;27(4):

417-418.

53. Weber JE, Bonzheim SC, Boczar ME, et al. The uncertain benefit of immediate stress testing (ETT) for low-intermediate risk patients with cocaine associated chest pain in the EDcardiac decision unit (CDU). Acad Emerg Med 2001;8:553.

54. Manini AF, Gisondi MA, van der Vlugt TM, et al. Adverse cardiac events in emergency department patients with chest pain six months after a negative inpatient evaluation for acute coronary syndrome. Acad Emerg Med 2002;9:896-902.

55. Kirk JD, Turnipseed S, Lewis WR, Amsterdam EA. Evaluation of chest pain in low-risk patients presenting to the emergency department: the role of immediate exercise testing. Ann Emerg Med 1998;32:1-7.

56. Schoepf UJ, Becker CR, Ohnesorge BM, et al. CT of coronary artery disease. Radiology 2004;232:18-37.

57. Hausleiter J, Meyer T, Hadamitzky M, et al. Radiation dose estimates from cardiac multislice computed tomography in daily practice impact of different scanning protocols on effective dose estimates. Circulation 2006;113:1305-1310.

58. Berman DS, Hachamovitch,R, Shaw LJ, et al. Roles of nuclear cardiology, cardiac computed tomography, and cardiac magnetic resonance: assessment of patients with suspected coronary artery disease. J Nucl Med 2006;47:74-82.

59. Dribben WH, Kirk MA, Trippi JA, et al. A pilot study to assess the safety of dobutamine stress echocardiography in the emergency department evaluation of cocaine-associated chest pain. Ann Emerg Med 2001;38(1):42-48. 